Chapter 8

Micro-Metrology 


\title{
TOWARDS A TRACEABLE INFRASTRUCTURE FOR LOW FORCE MEASUREMENTS
}

\author{
Richard K Leach, Christopher W Jones \\ Industry \& Innovation Division, National Physical Laboratory \\ Hampton Road, Teddington, Middlesex TW11 0LW \\ richard.leach@npl.co.uk
}

\begin{abstract}
Over the past ten years or so the need for the measurement of low forces ranging from newtons down to attonewtons has become increasingly important. As we begin to manufacture and manipulate structures on the micrometre to nanometre scale, the forces that are exerted in such processes must be controlled. To control such forces requires some form of measurement, either a direct measurement of the force, or a measurement of the effect the force has on the structure it is applied to. This paper is primarily concerned with the development of a traceability infrastructure for forces in the range from $1 \mathrm{nN}$ to $10 \mu \mathrm{N}$. The lower end of this force range does not cover chemical or most biological forces (usually in the femto- to piconewton range) despite the increasing importance of accurately measuring such forces. Further work is still required to push the limits of force traceability to these levels. At the upper end of the force range considered here, more traditional methods for measuring forces can be used that are traceable to the unit of mass, i.e. the force is realised as a mass in a gravitational field. The force range discussed in this paper applies to many nano- and micrometre scale manipulation and assembly applications, including micro-grippers, handlers and force feedback devices. Further applications that fall into the force range discussed here include the force exerted on a surface by atomic force microscopes and other scanning probe instruments, forces in the area of materials property measurement using indentation technology, the forces found in micro-electromechnical systems (MEMS) and the forces exerted by artificial biological tissues, for example muscle fibres. The two main force generation mechanisms that are found in nature and engineering are the weight of the mass of an object in a gravitational field and the deflection of an element with a finite spring constant. On the micro- to nanometre scale the spring force is more usually used to produce or react to a force, for example an AFM cantilever.
\end{abstract}

\section{Introduction}

Traceability for force measurement is usually carried out by comparing to a calibrated mass in a known gravitational field. However, as the forces (and hence masses) being measured decrease below around $10 \mu \mathrm{N}$ (approximately equivalent

Please use the following format when citing this chapter:

Leach, R. K., Jones, C. W., 2008, in IFIP International Federation for Information Processing, Volume 260, MicroAssembly Technologies and Applications, eds. Ratchev, S., Koelemeijer, S., (Boston: Springer), pp. 307-314. 
to $1 \mathrm{mg}$ ), the uncertainty in the mass measurement becomes too large and the masses become difficult to handle. For this reason it is more common to have a force balance that gains its traceability through electrical and length measurements.

The current force traceability route is at least a two-stage process. The first stage is to develop a primary force standard instrument deriving traceability directly from the base unit definitions realised at the world's National Measurement Institutes (NMIs). These primary instruments will typically sacrifice practicalities in order to obtain the best possible metrological performance. Various groups have developed such instruments, with the current best performance held by examples at the $\mathrm{Na}$ tional Institute of Science and Technology (NIST) in the USA and the National Physical Laboratory (NPL) in the UK.

The second stage in the traceability route is to design a transfer artefact, or sequence of artefacts, to transfer the force calibration to target instruments in the field. These artefacts may sacrifice uncertainties, resolution or range of force measurement, in exchange for cost reductions, portability or compliance with other physical constraints, such as size or environmental tolerance.

\section{Primary Force Balances}

The leading examples of force measurement instruments are based on the electrostatic force balance principle. The force to be measured is exerted on a flexure system, which deflects. This deflection is interferometrically determined. The deflection of the flexure also changes the capacitance of a set of parallel capacitor plates in the instrument. This is usually achieved either by changing the plate overlap, or by changing the position of a dielectric, with flexure deflection. In this way the capacitance changes linearly with deflection. The interferometer signal is used in a closed-loop controller to generate a potential difference across the capacitor generating an electrostatic force that servos the flexure back to zero deflection. Measurement of the force exerted is derived from traceable measurements of length, capacitance and potential difference. The exerted force is calculated using equation 1 , in which $z$ is the flexure displacement, and $C$ and $V$ the capacitance of and voltage across the parallel plates respectively. The capacitance gradient, $\mathrm{d} C / \mathrm{d} z$, must be determined prior to use and is a source of statistical uncertainty that must be quantified. Uncertainties are associated with external system interaction, as well as with the measurement of $z, V$ and $C$, and with contributions from flexure misalignment, corner loading and hysteresis.

$$
F=-\frac{1}{2} V^{2} \frac{\mathrm{d} C}{\mathrm{~d} z}
$$

\section{I The NIST Electrostatic Force Balance}

NIST's Electrostatic Force Balance (EFB) [1] was the first major example of a fully traceable primary low force balance. The NIST EFB, which is currently operated in 
a vacuum, has a working range of $10 \mathrm{nN}$ to $1 \mathrm{mN}$ with accuracy of parts in $10^{4}$ and a sub-nanonewton resolution [2]. The instrument, schematically shown in Figure 1, is currently being used to characterise prototype transfer artefacts. The capacitor system is created from two coaxial cylinders, the insertion of one inside the other varied with balance deflection leading to the desired linear capacitance gradient.

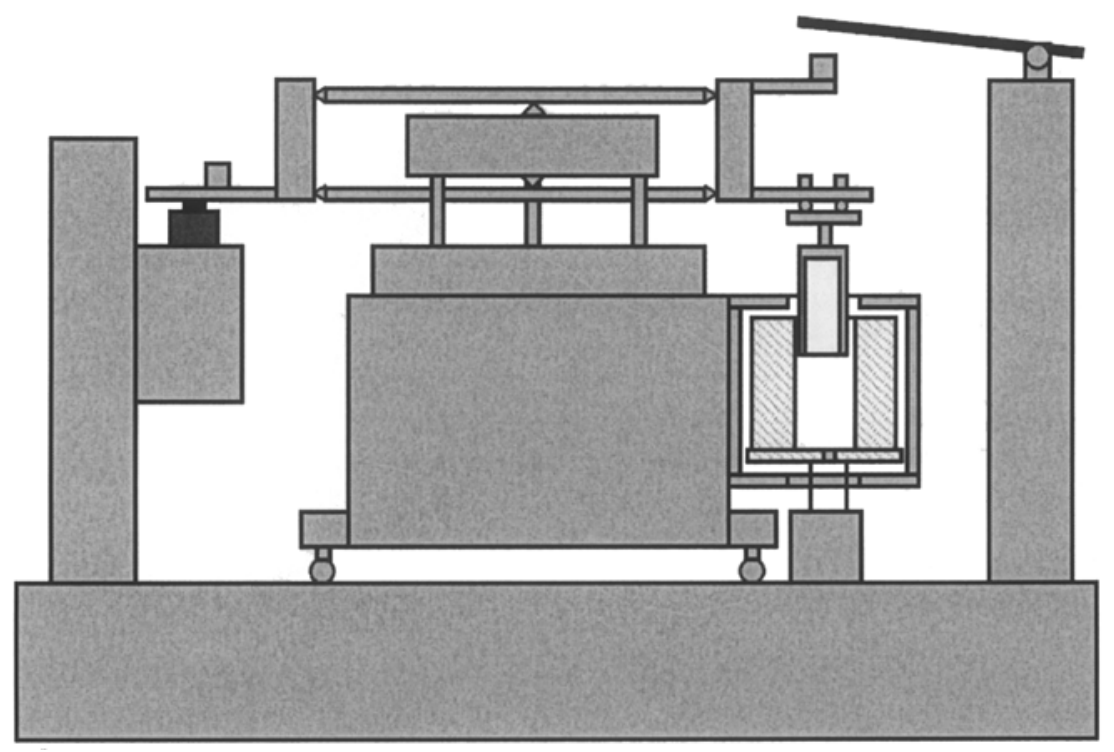

Fig. 1. Schematic diagram of the NIST EFB, from [2]. The hatched item on the right is the outer capacitor cylinder, with the inner cylinder suspended from the flexure system across the top. The red electromagnetic actuator moves the balance for $\mathrm{d} C / \mathrm{d} z$ determination; the black line shown in the top right corner is a mass loader for wire test masses for the higher force range.

\subsection{The KRISS Electrostatic Force Balance}

The Korea Research Institute of Standards and Science (KRISS) also has plans in place to construct a low force balance. Much of their effort so far has been concerned with modelling of the motion of the basic low force balance parallelogram flexure arrangement, focussing on the dependency of good results on parallelism and mechanisms to fine-tune the geometry of the flexure [3].

\subsection{The NPL Low Force Balance}

The unique, monolithic construction of the flexure arrangement in the NPL Low Force Balance (NPL LFB) $[4,5]$ combined with its smaller size promises better low-end force measurement performance than its current international equivalents. The instrument's range is $1 \mathrm{nN}$ to $10 \mu \mathrm{N}$ with a design resolution of $50 \mathrm{pN}$. The 
NPL LFB, schematically shown in figure 2, was designed and constructed under contract by the Technical University Eindhoven [6]. Displacement of a dielectric blade in the capacitor, connected to the flexure, is measured using a plane mirror differential interferometer. The feedback voltage generates a force on the blade that returns the flexure displacement to zero. The NPL LFB is currently undergoing characterisation work and preliminary results will be presented shortly.

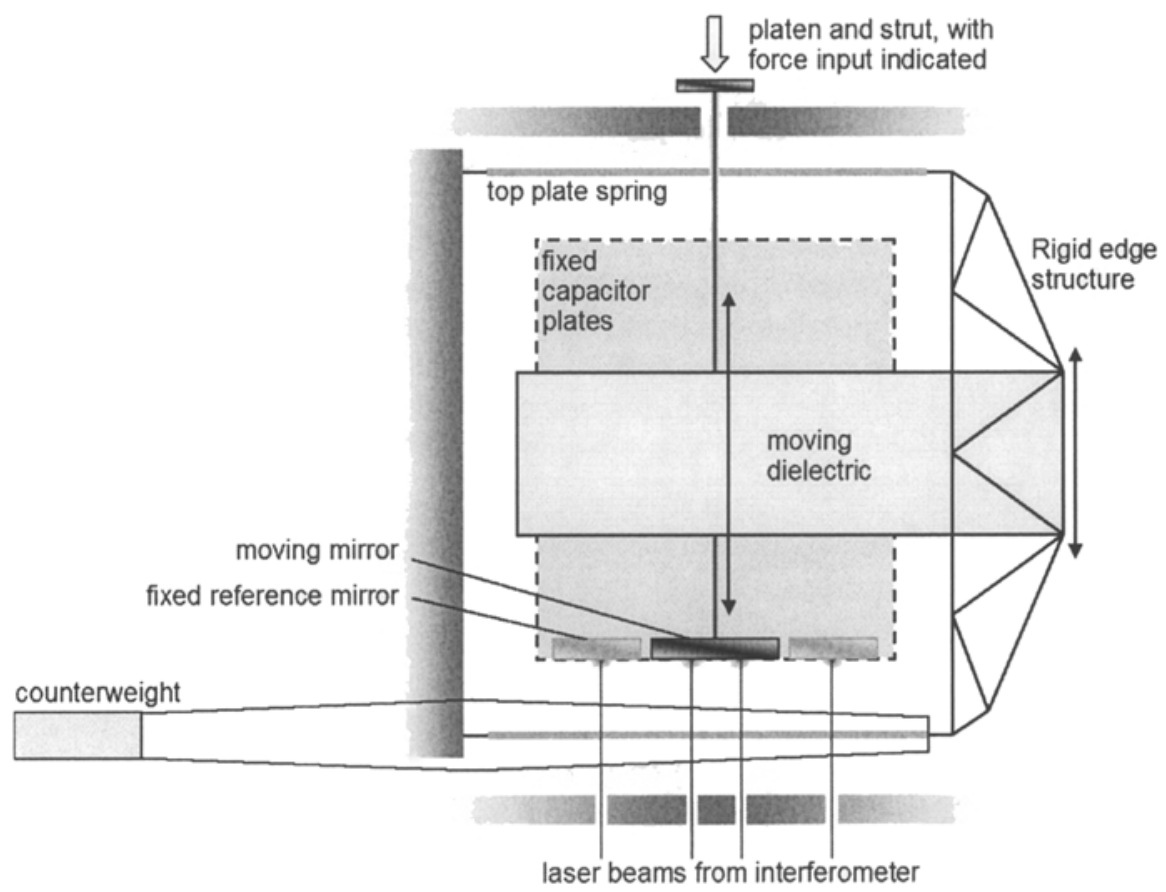

Fig. 2. Schematic diagram of the NPL LFB working mechanism. Force exerted on the platen deflects the dielectric vertically against the plate springs, with motion constrained by the rigid edge structure. The flexure, including the edge structure and solid supporting frame, were monolithically manufactured. The counterweight can accept additional loads to offset test masses. The spring constant of the balance is around $1 \mathrm{~N} \mathrm{~m}^{-1}$.

\subsection{High-end performance: deadweight measurement}

An important partial verification of the performance of a low force balance occurs at the overlap of the operating ranges of the deadweight calibration system and the balance. This has been carried out successfully [7] on the larger NIST EFB, which has a relatively high upper force range limit allowing larger and more practical test masses. The significantly lower upper force limit of the NPL LFB adds to the complexity of mass intercomparison with the balance's electrostatic force measurements. Efforts are underway at NPL to produce a suite of low mass arte- 
facts using novel materials that may be calibrated and handled such that relative uncertainties are significantly reduced. Calibrated on the NPL primary mass balance, these will be weighed on both the NPL Low Force Balance and NIST EFB.

\subsection{Low-end performance: radiation pressure experiments}

Verification of the lower end of the low force balance's force range may be obtained by comparison with radiation pressure, adapting similar examples (such as [7]). A medium power laser would be directed onto the end of the balance platen, to which a very high reflectivity ( $>99.99 \%$ ) mirror is attached, of suitable size to catch all of the laser light. Assuming the ideal case with no losses, a nanonewton of force would be generated by 150 milliwatts of input optical power. Care would need to be taken to eliminate spurious radiometric effects, which would need to be traded off with lower heat dissipation when deciding on the use of a vacuum for operation. Some noise will originate from the kinematic effect of particles excited off the mirror. The design of the NPL LFB combined with operation in position-nulled mode is such that thermal expansion in the vicinity of the platen would have a negligible effect on the measured force. Furthermore, with a high-reflectivity mirror in place the transmitted power would be of the order of a milliwatt or less, acceptable provided exposure times are kept short.

\section{Low force transfer artefacts}

A number of groups worldwide have worked to develop technologies that may be implemented as low force transfer artefacts. These projects were motivated by different industrial needs and, therefore, vary in force range, tolerance to environment and target instrument. It is the task of the NMIs in collaboration with industry to develop working transfer artefacts.

\subsection{Springs}

After gravitational forces from calibrated masses the most intuitive and common technology used for calibrated force production is an elastic element with a known spring constant. The element, such as a cantilever or helical spring, is deflected by a test force. The deflection is measured, either by an external system such as an interferometer, or by an on-board MEMS device such as a piezoelectric element. With the spring constant previously determined by a traceable instrument such as an electrostatic force balance, the size of the test force can be calculated. Recent examples of artefacts use modified AFM cantilevers with piezoresistive deflection measurement, as shown in Figure 3. These examples also have fiducial markings along their length to reduce a major source of uncertainty for this type of elastic element device, the position of the artefact-target interaction. Effective spring constants are known for several positions along the length of the cantilevers, allowing calibration 
transfer in a number of ranges. An alternative approach, also developed at NPL, is a MEMS device known as MARS (Microfabricated Array of Reference Springs). The device consists of a number of reflective discs supported on helical springs of different nominal spring constants [9]. Lower sensitivity to test probe interaction position is a distinct advantage of this type of spring device but the added complexity of the elastic system makes it harder to model and evaluate.

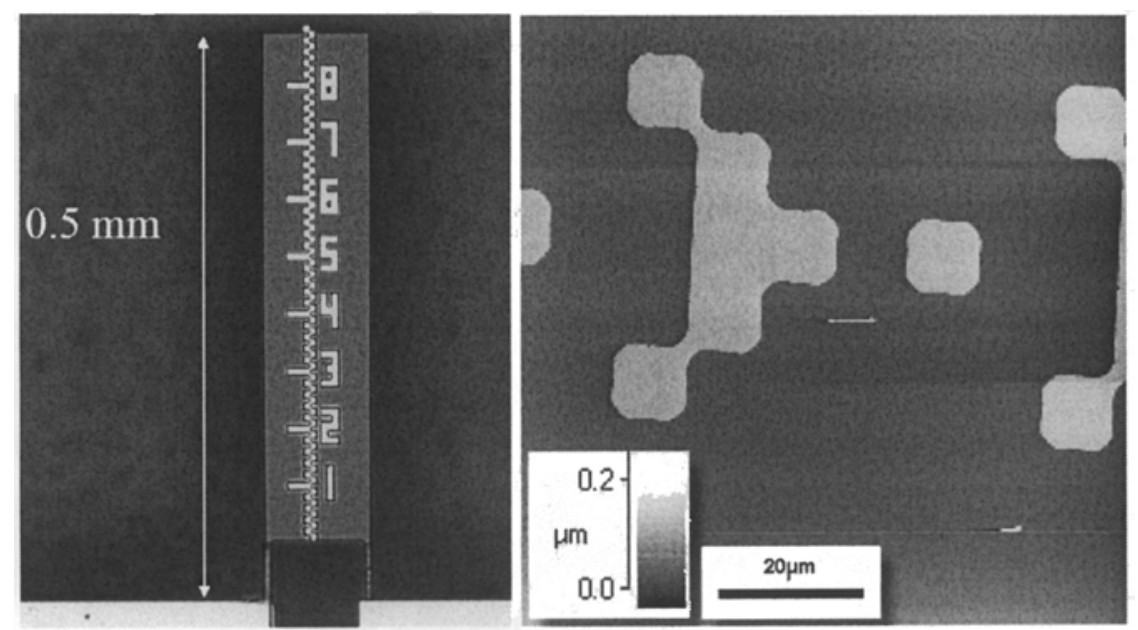

Fig. 3. (left) the NIST (from Pratt 2007); (right) detail of the fiducial markings on the NPL CMARS device; the $10 \mu \mathrm{m}$ squares form a binary numbering system along the axis of symmetry (from Cumpson et al 2004 [10]).

\subsection{Resonant structures}

Alternative propositions take advantage of the noise advantages of frequency modulation. Changes in the tension of a stretched string can be detected via related changes in its resonant frequency. If a force is exerted on one of the string anchor points along the string axis, the tension in the string will decrease. For a wellcharacterised string the force exerted can be calculated from an accurate determination of the frequency shift. In this way a low-force measurement device is created. With a careful choice of working frequency through device design, the effect of noise on the measurement signal can be dramatically reduced in comparison to other methods. Stalder and Dürig's 'nanoguitar' [12, shown in Figure 4, is one example of such a device. Similar devices resembling double-ended tuning forks replace the string with narrow plate oscillators [12]. The challenge for resonance methods is in making the frequency measurement system, usually optical interrogation by laser, robust and compact enough for use in a transfer artefact. 


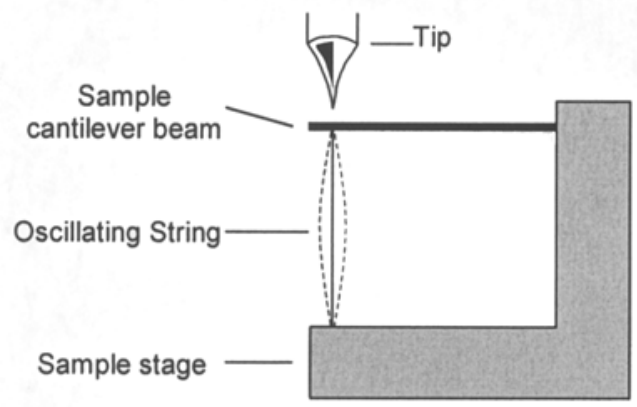

Fig. 4. Schematic of a resonant string sensor (after [10])

\section{The future - intrinsic force standards}

Completion of the traceability link at the micro- and nanonewton scale will see development efforts shifting towards even smaller forces. Traceability at the levels fundamental to biochemistry, such as covalent bonds $(100 \mathrm{pN})$ and thermal motion of DNA molecules $(100 \mathrm{fN})$ is now required, for example to verify theoretical models that may be inaccurate.

Further radiation pressure experiments may present a possibility, as may the electrostatic force between a probe and a surface of well-defined geometry.

It is hoped that at this scale it will be possible to develop intrinsic force standards based, for example, on reversible conformation changes in proteins. To date results have been mixed. Well-defined force steps in the $100 \mathrm{pN}$ to $300 \mathrm{pN}$ range, as protein structures are unfolded in sequence, have been reported [13]. However extreme hysteresis has been observed when reversing such extensions in similar experiments [14].

As collaborative efforts are developed between biochemical institutions and traditional engineering metrologists significant advances of force traceability into the piconewton range should be made.

\section{References}

1. J. R. Pratt, D. T. Smith, D. B. Newell, J. A. Kramar and E. Whitenton, Progress toward Système International d'Unités traceable force metrology for nanomechanics, J. Mat. Res. 19, 366-379 (2004)

2. J. R. Pratt, private correspondence (2007)

3. I.-M. Choi, M.-S. Kim, S.-Y. Woo and S. H. Kim, Parallelism error analysis and compensation for micro-force measurement, Meas. Sci. Technol. 15, 237-243 (2004)

4. R. K. Leach, S. Oldfield, S. Awan, J. Blackburn and J. M. Williams, Design of a bidirectional electrostatic actuator for realising nanonewton to micronewton forces, NPL Report DEPC-EM-001, 1-32 (2004) 
5. R. K. Leach, S. Oldfield, D. Georgakopulos, Traceable nanonewton force measurement at the National Physical Laboratory, UK, Proc. $6^{\text {th }}$ euspen Int. Conf., Baden, Austria, May, 414-417 (2006)

6. R. Henselmans, N. Rosielle, L. Cacace, P. Kappelhof, F. Klinkhamer and H. Spierdijk, Low force measurement facility: mechanical design report (Technical University of Eindhoven) (2004)

7. D. B. Newell, J. A. Kramar, J. R. Pratt, D. T. Smith and E. R. Williams, The NiST Microforce Realization and Measurement Project, IEEE Transactions on instrumentation and measurement 52, 508-511 (2003)

8. M. Feat, C. Zhao, L. Ju and D. G. Blair, Demonstration of low power radiation pressure actuation for control of test masses, Rev. Sci. Instr. 76, 036107 (2005)

9. P. J. Cumpson, J. Hedley and P. Zhdan, Accurate force measurement in the atomic force microscope: a microfabricated array of reference springs for easy cantilever calibration, Nanotechnology 14, 918-924 (2003)

10. P. J. Cumpson, C. A. Clifford and J. Hedley, Quantitative analytical atomic force microscopy: a cantilever reference device for easy and accurate AFM spring-constant calibration, Meas. Sci. Technol. 15, 1337-1346 (2004)

11. A. Stalder and U. Dürig, Nanoguitar: oscillating string as force sensor, Rev. Sci. Instrum. 66, 3576-3579 (1995)

12. K. Fukuzawa, T. Ando, M. Shibamoto, Y. Mitsuya and H. Zhang, Monolithically fabricated double-ended tuning-fork-based force sensor, J. Appl. Phys. 99, 094901 (2006)

13. M. Rief, M. Gautel, F. Oesterhelt, J. M. Fernandez, H. E. Gaub, Reversible unfolding of individual titin immunoglobulin domains by AFM, Science, 276, 1109-1112 (1997)

14. A. Oberhauser, P. Hansma, M. Carrion-Vazquez and J. M. Fernandez, Stepwise unfolding of titin under force-clamp atomic force microscopy, PNAS 98, 468-472 (2001) 